The Astrophysical Journal, 679: L53-L56, 2008 May 20

(C) 2008. The American Astronomical Society. All rights reserved. Printed in U.S.A.

\title{
NO EVIDENCE OF MORNING OR LARGE-SCALE DRIZZLE ON TITAN
}

\author{
Sang J. Kim, ${ }^{1}$ Laurence M. Trafton, ${ }^{2}$ and Thomas R. Geballe ${ }^{3}$ \\ Received 2008 February 19; accepted 2008 March 25; published 2008 April 23
}

\begin{abstract}
Widespread methane precipitation on Titan has been predicted by several authors since methane saturation in the upper troposphere was measured by the Huygens probe over its landing site. Recently, observational evidence of widespread morning drizzle has been suggested based on detection of a dark morning site in surface-subtracted $2 \mu \mathrm{m}$ spectroimages obtained from ground-based observations. We have obtained similar new spectroimages of Titan observed at Gemini-North Observatory at several rotational phase angles and have analyzed them using the same image processing technique. By comparing images at different phases, we show that the presumed dark drizzling regions in the surface-subtracted images are mainly negative renditions of bright surface regions. No evidence of spectroscopically verifiable drizzling is apparent in our cloudless Titan images. At present, constraining models of Titan's hydrological cycle to produce a diurnal response would be premature.
\end{abstract}

Subject headings: infrared: solar system — planets and satellites: individual (Titan) — solar system: general

\section{INTRODUCTION}

Titan's hydrology is a major science focus of the Cassinil Huygens missions (e.g., Barnes et al. 2007; Griffith et al. 2005), and of ground-based telescopic observations (Brown et al. 2002; Roe et al. 2005). Whether atmospheric drizzle occurs on Titan and whether it is a periodic (e.g., daily) phenomenon are important questions that are relevant not only to understanding the specific processes and mechanism of drizzle formation, but also to understanding weather in general on Titan (Courtin et al. 1995; Tokano et al. 2006; Ádámkovics et al. 2007). Persistent clouds in the polar regions and intermittently occurring clouds in the southern temperate regions of Titan have been observed by the Visual and Infrared Mapping Spectrometer (VIMS) on Cassini (Barnes et al. 2007; Griffith et al. 2005), and some of these have also been observed before Cassini from ground observatories through near-IR atmospheric windows (Brown et al. 2002; Roe et al. 2005). Indeed, tropospheric saturation and consequent methane rainfall have been hypothesized since the Voyager 1 and 2 encounters with Titan (Courtin et al. 1995). Based on the vertical methane humidity profile measured over the Huygens landing site, drizzle on a global scale has been suggested (Tokano et al. 2006).

Recently, it has been suggested that widespread morning drizzle occurs on Titan based on an analysis of $2 \mu \mathrm{m}$ spectroimages from observations at the Very Large Telescope (VLT) in Chile and the W. M. Keck Observatories in Hawaii (Ádámkovics et al. 2007). This analysis involved examination of surface-subtracted images $(\Delta I / F)$ constructed using a simple linear equation: $\Delta I / F=I_{2} / F-f \times I_{1} / F$, where the $I_{2} / F$ are lower tropospheric images at 2.060-2.070 $\mu \mathrm{m}$, similar to those images in Figure $1 b$; the $I_{1} / F$ are surface images at 2.027$2.037 \mu \mathrm{m}$, similar to those images in Figure $1 a$; and the $f$ are scaling factors. Titan's atmosphere in the $2.027-2.037 \mu \mathrm{m}$ spectral range is thought to be sufficiently optically thin to reveal primarily surface features, whereas in the 2.060-2.070 $\mu \mathrm{m}$ range, for which Titan's spectral continuum is lower than that at 2.027-2.037 $\mu \mathrm{m}$ (Fig. 2a), its spectrum could be significantly affected by absorption from haze and/or methane drizzle

\footnotetext{
${ }^{1}$ Department of Astronomy and Space Science, Kyunghee University, Yongin 446-701, Korea.

${ }^{2}$ Department of Astronomy, University of Texas, Austin, TX 78712.

${ }^{3}$ Gemini Observatory, 670 North A'ohoku Place, Hilo, HI 96720.
}

(Grundy et al. 2002). The value of $f$ was determined by the condition that the cross-correlation of the surface-subtracted image intensity $(\Delta I / F)$ with the surface image $(2.027-2.037$ $\mu \mathrm{m}$ ) be a minimum when evaluated over the disk (Ádámkovics et al. 2007). For this value, the supposition is that if the Titanian haze is uniformly distributed over the disk, haze effects should be removed and other nonuniform tropospheric opacity sources, such as drizzle, would be revealed in the $\Delta I / F$ images. Using this simple logic, it has been suggested that the dark regions appearing in the $\Delta I / F$ images of Figure $3 a$ are caused by absorption from morning drizzle (Ádámkovics et al. 2007). However, as discussed below, applying an $f$ value that corresponds to minimum correlation over all the pixels on the disk image can cause an over- or undersubtraction for relatively bright or dark local regions of Titan's surface, for surface regions having differing spectral slopes, or for regions partially obscured by haze.

\section{OBSERVATIONS}

In order to test the validity of this empirical approach, we analyze similar but independent spectroimages and spectra of Titan (Figs. $1 a$ and $1 b$, and Fig. $2 a$ ) obtained using the NearInfrared Integral Field Spectrometer (NIFS) (McGregor et al. 2003) on the Gemini-North telescope in Hawaii with adaptive optics, which maintained an effective spatial resolution of $0.1^{\prime \prime}$ over Titan's $0.9^{\prime \prime}$ diameter disk. Each pixel sampled a rectangular region of sky of dimension 0.1" parallel to Titan's disk equator by $0.04^{\prime \prime}$ perpendicular to it. The images were interpolated and resampled to pixels $0.05^{\prime \prime}$ square shown in the figures. The spectral resolving power was $\sim 5000$ and image planes were sampled in wavelength every $2.13 \AA$. The telluric spectrum, which is dominated by $\mathrm{CO}_{2}$ lines in this wavelength region, was removed by dividing by the spectrum of a solartype star; however, the weak solar lines were not perfectly canceled owing to the star's Doppler shift. The count rate was corrected for air mass but a flux standard star was not observed. As no clouds were apparent on Titan at the time of our observations, the $I / F$ calibration was based on the relative intensities normalized to known albedos of cloudless Titan at $2 \mu \mathrm{m}$ (Griffith et al. 1998, 2000). Titan's rotation period is 16 days; data were obtained for three different longitude regimes by observing Titan on three different nights. 


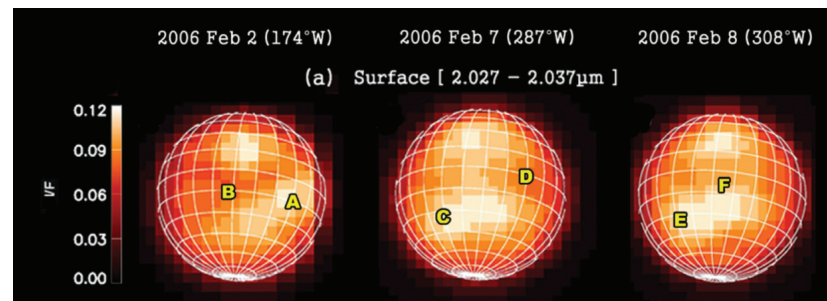

(b) Lower troposphere [ $2.060-2.070 \mu \mathrm{m}]$
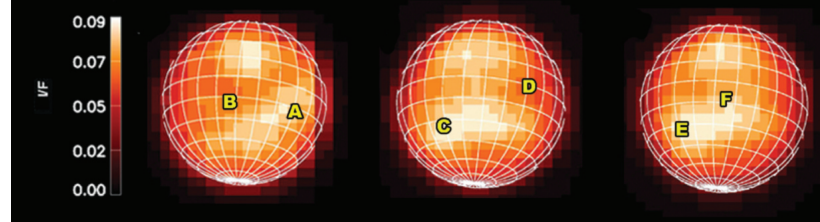

(c) Barnes et al. (2008)
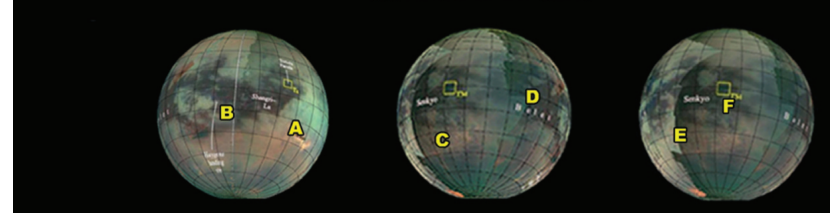

FIG. 1.-Shown are $2 \mu \mathrm{m}$ images of Titan observed on 2006 February 2, 7 , and 8 with NIFS on Gemini when no clouds were visible except in the south polar region. (a) The $I / F$ images in the first row were made by summing spectroimage cubes between 2.027 and $2.037 \mu \mathrm{m}$, a spectral window through which the surface features are thought to be revealed clearly (Ádámkovics et al. 2007). (b) The second-row $I / F$ images were made by summing up spectroimages between 2.060 and $2.070 \mu \mathrm{m}$, a range over which absorptions by haze and possible methane drizzle are influential (Ádámkovics et al. 2007). (c) The third-row images made from a Mercator map of VIMS observations (Barnes et al. 2008) correspond to the views from Earth at the times of the Gemini NIFS observations. The sharp discontinuities in the $I / F$ images are artifacts of the mosaicking. Despite the limb darkening shown in the NIFS images, major features (marked by A, B, C, D, E, and F) on the NIFS images are shown to occur at the same places on the VIMS images.
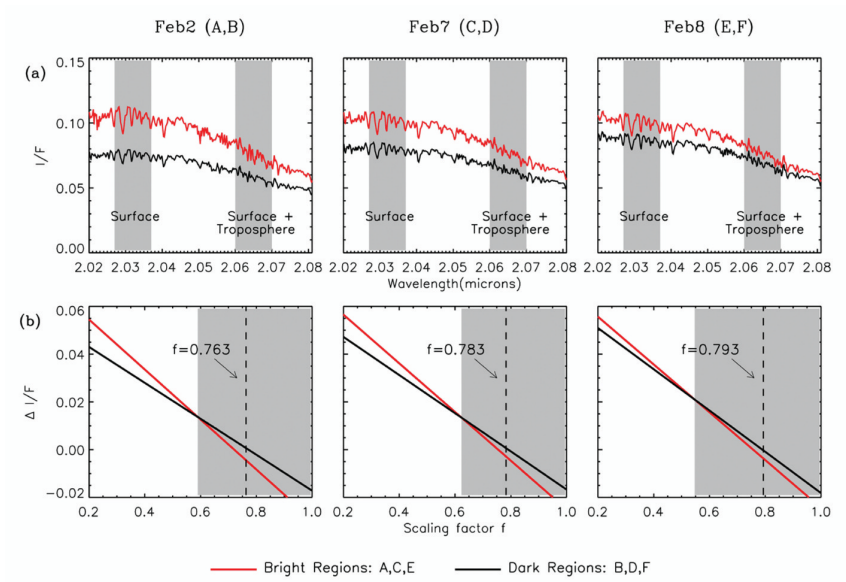

FIG. 2.-(a) The spectra in the top panels span the spectral ranges of the NIFS spectroimages obtained on 2006 February 2, 7, and 8, respectively, with a spectral resolving power of $\sim 5000$. In each panel, two spectra taken from bright and dark regions marked by capital letters in Fig. 1 are presented for comparison. The spectral ranges from where presumably the surface and surface + tropospheric images were taken are marked by vertical shadows. (b) The bottom panels show the different degrees of dependence on the surface image scaling factor $f$ of the solutions of two linear equations that attempt to remove the surface influence from the surface+tropospheric image (see text). The two equations correspond to the intensity over two representative regions, one bright and the other dark. The intersection of these lines marks values of $f$ above which (shaded areas) the contrast of these surface features is inverted in the surface-subtracted difference image $(\Delta I / F ;$ Fig. $3 b)$. The scaling factors $f$ obtained at minimum correlations between $\Delta I / F$ and surface images (Fig. 1a) are marked with vertical dashed lines, which lie well into the shaded (inversion) regime. These graphs demonstrate that an application of such an $f$ value to all the pixels in an image cannot completely eliminate surface features and may create the anticorrelated surface features in the $\Delta I / F$ image shown in Fig. $3 a$.

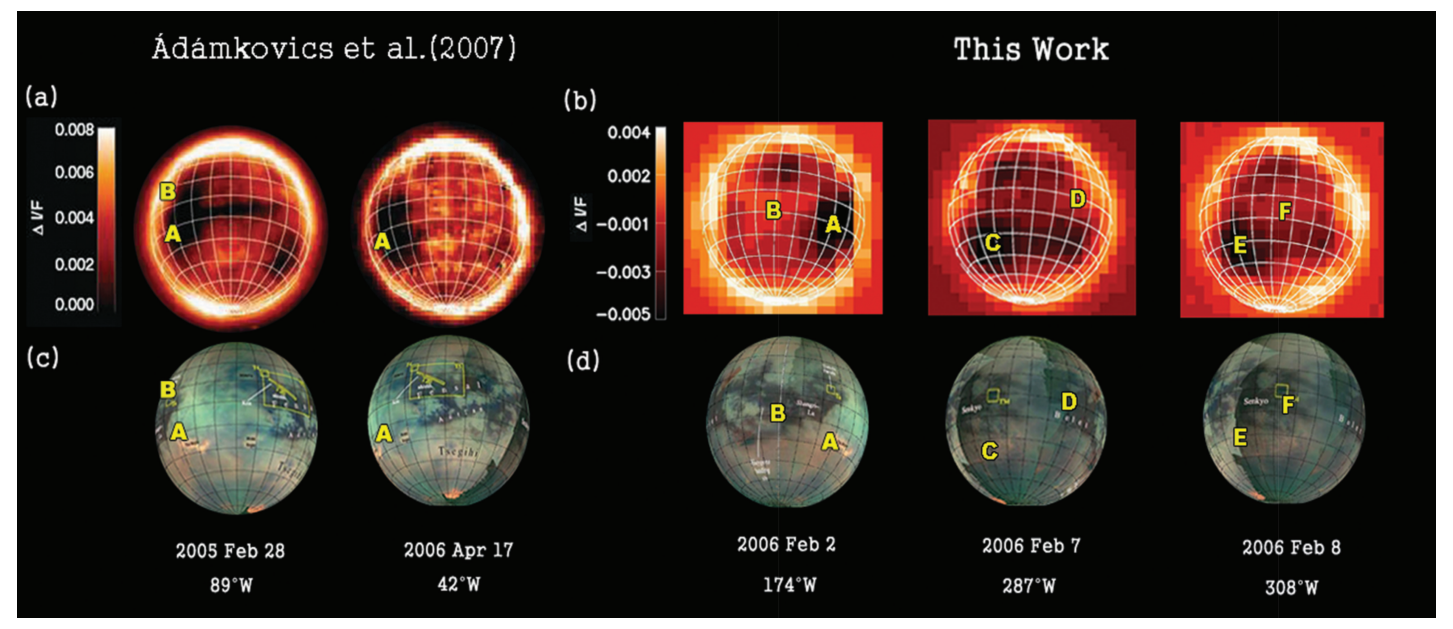

FIG. 3.- $(a, b)$ Nominally surface-subtracted images $(\Delta I / F)$ compared with $(c, d)$ corresponding VIMS images viewed from Earth at the times of the ground observations. The dark region (A) shown in (a) has been suggested to be an area of morning drizzling (Ádámkovics et al. 2007), which largely corresponds to Xanadu and nearby bright areas in the VIMS images. However, this dark region is also identified in our image $(b)$, but in the late afternoon. The bright region (B) in the $\Delta I / F$ images is identified as a relatively dark region, Shangri-La, in the VIMS images. This kind of approximate anticorrelation is also present in the other $\Delta I / F$ images and corresponding VIMS images at different longitudes. No distinctive dark regions in the $\Delta I / F$ images other than the gross anticorrelations with the VIMS surface features are found, indicating that no spectroscopically verifiable widespread drizzle is apparent in the $2 \mu \mathrm{m}$ images, although drizzle might actually be occurring over small areas or very lightly over large regions. 


\section{IMAGE PROCESSING AND ANALYSIS}

We derived $\Delta I / F$ images (Fig. $3 b$ ) at three different subEarth longitudes using the same techniques as used for the VLT and Keck data (Ádámkovics et al. 2007), employing the following equations:

$$
\Delta I_{i} / F=I_{i 2} / F-f I_{i 1} / F
$$

where $i$ is a pixel on the disk and 1 and 2 are for the 2.0272.037 and 2.060-2.070 $\mu \mathrm{m}$ ranges, respectively. $I_{i 1}$ and $I_{i 2}$ are obtained by averaging $I_{i}$ within the above wavelength ranges. Using statistical correlative analyses, we found three different $f$ values of $0.763,0.783$, and 0.793 (vertical lines in Fig. $2 b$ ) for 2006 February 2, 7, and 8, respectively, at which each $\Delta I / F$ image has a minimum correlation with each surface (2.027-2.037 $\mu \mathrm{m}$ ) image (Fig. $1 a$ ). The resulting $\Delta I / F$ images, in which the surface features are presumably eliminated, are presented in Figure $3 b$. We also present virtual Titan disks (Fig. $1 c$, and Figs. $3 c$ and $3 d$ ) based on a Mercator map from VIMS observations (Barnes et al. 2008) to compare large-scale surface features shown in the NIFS images with those shown in the VIMS images.

The major features shown in the $\Delta I / F$ images in Figure 3 are approximately anticorrelated with surface features in the VIMS disk images. For example, the bright region known as Xanadu and marked A in Figure $3 b$ turns out to be dark in the $\Delta I / F$ image (Fig. $3 a$ ), while the dark region Shangri-La, marked $\mathrm{B}$ in Figure $3 b$, turns out to be bright in the $\Delta I / F$ image. This kind of anticorrelation between the $\Delta I / F$ images and corresponding VIMS images at the same longitudes is also evident in the remainder of Figure 3. In particular, the dark region in the $\Delta I / F$ image (marked $\mathrm{A}$ in Fig. $3 a$ ), which has been suggested to be a morning drizzling area, turns out to be the same dark region on the late afternoon site in our 2006 February 2 image (marked A in Fig. 3b).

The gross anticorrelations can be explained as graphically shown in $\Delta I / F$ and $f$ space (Fig. $2 b$ ) using the following equations:

$$
\begin{aligned}
& \Delta I_{b} / F=I_{b 2} / F-f I_{b 1} / F, \\
& \Delta I_{d} / F=I_{d 2} / F-f I_{d 1} / F,
\end{aligned}
$$

where $b$ and $d$ are for representative bright and dark regions marked in the figures. The inversion (shaded parts in Fig. $2 b$ ) from the bright to dark regions or dark to bright regions in the $\Delta I / F$ images begins well below the minimum correlation $f$ values marked as vertical lines in Figure $2 b$. These graphs demonstrate that an application of such a representative $f$ value to all the pixels in an image cannot eliminate surface features entirely and may create false or misleading signatures, such as anticorrelated or negative surface features, in the $\Delta I / F$ image. In addition, the following two difficulties arise when we use the empirical subtraction approach. First, because spectral slopes for different terrains on Titan's surface may not be the same (McCord et al. 2006), simple subtraction using a scaling factor $f$ may also yield local image features arising primarily from the spectral differences among terrains instead of primarily from albedo differences or from purely tropospheric properties. Second, the scaled subtraction equation neglects any spatial variation in the multiple-scattering of haze. The haze distribution over Titan's disk may not be uniform (de Kok et al. 2007), in which case the scaled subtraction would simply highlight the haze variation over the disk.

If a high spatial resolution model of Titan's surface reflectivity were available at these wavelengths and if multiple scattering by haze were included, it might be possible to isolate the effects of drizzle and test for its presence. For now we conclude that considerably more information about Titan's surface and the properties of its haze is needed before drizzle can be differentiated from local anticorrelations.

\section{CONCLUSIONS}

Since we could not find any distinctive dark regions in our $\Delta I / F$ images obtained at different longitudes other than the approximately anticorrelated or inverted features, we conclude that there is no apparent evidence in the $2 \mu \mathrm{m}$ spectroimages for widespread drizzle that is spectroscopically verifiable. Moreover, we conclude that there is so far no spectroscopic evidence that drizzle is a morning phenomenon on Titan. It would therefore be premature to constrain models of Titan's hydrological cycle to produce such a diurnal response. Small active drizzling areas might exist and escape detection, as could sufficiently light drizzle over larger areas.

There seems to be no easy way to isolate the spectral signature of drizzle in these $2 \mu \mathrm{m}$ spectroimages. Titanian drizzle can be remotely sensed using rigorous radiative transfer analysis only after the $2 \mu \mathrm{m}$ spectral characteristics of different terrains and scattering properties of haze particles and clouds are accurately known, from, e.g., ongoing detailed investigations based on CassinilHuygens data. Although monitoring Titanian rainfall from Earth is attractive, it is additionally difficult, even if the above are known, because high signal-to-noise ratio spectroimages are required to extract the subtle differences in the images that might isolate the effects of absorption by drizzle from, e.g., temporal variations in the spatial distribution and resulting amount of multiscattering by haze. Additional data, such as a time series of spectroimages, are needed for investigating under what conditions the signature of drizzle can be extracted unambiguously from remotely sensed Titanian images.

The authors thank A. Jung, H. Seo, and C. Sim for rendering the figures and performing image processing. L. M. T. acknowledges support from NASA grant NNG04G131GGR. We thank the two referees, whose constructive comments improved the manuscript. Based on observations (GN-2006A-SV-128) obtained at the Gemini Observatory, which is operated by the Association of Universities for Research in Astronomy, Inc., under a cooperative agreement with the NSF on behalf of the Gemini partnership: the National Science Foundation (United States), the Science and Technology Facilities Council (United Kingdom), the National Research Council (Canada), CONICYT (Chile), the Australian Research Council (Australia), CNPq (Brazil), and SECYT (Argentina). 


\section{REFERENCES}

Ádámkovics, M., Wong, M. H., Laver, C., \& de Pater, I. 2007, Science, 318, 962

Barnes, J. W., et al. 2007, Icarus, 186, 242

2008, Icarus, in press (doi: 10.1016/j.icarus.2007.12.006)

Brown, M. E., Bouchez, A. H., \& Griffith, C. A. 2002, Nature, 420, 795

Courtin, R., Gautier, D., \& McKay, C. P. 1995, Icarus, 114, 144

de Kok, R., et al. 2007, Icarus, 191, 223

Griffith, C. A., Hall, J. L., \& Geballe, T. 2000, Science, 290, 509
Griffith, C. A., Owen, T., Miller, G. A., \& Geballe, T. 1998, Nature, 395, 575 Griffith, C. A., et al. 2005, Science, 310, 474

Grundy, W. M., Schmitt, B., \& Quirico, E. 2002, Icarus, 155, 486

McCord, T. B., et al. 2006, Planet. Space Sci., 54, 1524

McGregor, P., et al. 2003, Proc. SPIE, 4841, 1581

Roe, H. G., Brown, M. E., Schaller, E. L., Bouchez, A. H., \& Trujillo, C. A. 2005, Science, 310, 477

Tokano, T., McKary, C. P., Neubauer, F. M., Atreya, S. K., Ferri, F., Fulchignoni, M., \& Niemann, H. B. 2006, Nature, 442, 432 